\title{
BeppoSAX, the wide band mission for X-ray astronomy
}

\author{
G. Boella ${ }^{1,7}$, R.C. Butler ${ }^{2}$, G.C. Perola ${ }^{3}$, L. Piro ${ }^{4}$, L. Scarsi ${ }^{5}$, and J.A.M. Bleeker ${ }^{6}$ \\ 1 CNR - Istituto di Fisica Cosmica e Tecnologie Relative, Via Bassini 15, 20133 Milano, Italy \\ 2 Agenzia Spaziale Italiana, Viale Regina Margherita 202, 00198 Roma, Italy \\ 3 Dipartimento di Fisica, Universita' Roma 3, Via Vasca Navale 84, 00146 Roma, Italy \\ ${ }^{4}$ CNR - Istituto di Astrofisica Spaziale, Via E. Fermi 21, 00044 Frascati, Italy \\ ${ }^{5}$ CNR - Istituto di Fisica Cosmica ed Applicazioni Informatica, Via Mariano Stabile 172, 90139 Palermo, Italy \\ 6 Space Research Organization of the Netherlands, Sorbonnelaan 2, 3584 CA Utrecht, The Netherlands \\ 7 Dip. Fisica, Universita' di Milano, Via Celoria 16, 20133 Milano, Italy
}

Received May 15; accepted October 21, 1996

\begin{abstract}
The X-ray satellite SAX, a major program of the Italian Space Agency with participation of the Netherlands Agency for Aereospace Programs, was launched on April 301996 from Cape Canaveral. After launch it was renamed BeppoSAX in honour of Giuseppe (Beppo) Occhialini. The payload is characterized by a very wide spectral coverage from 0.1 to $300 \mathrm{keV}$, with well balanced performances both from its low and high energy instrumentation. Its sensitivity will allow the exploitation of the full band for weak sources $(1 / 20$ of $3 \mathrm{C} 273)$, opening new perspectives in the study of spectral shape and variability of several classes of objects. Furthermore, the presence of wide field cameras will allow monitoring of the long term variability of sources down to $1 \mathrm{mCrab}$ and the discovery of X-ray transient phenomena. In this paper we describe the main aspects of the mission, the operations, the scientific capabilities of the instruments and the scientific objectives.
\end{abstract}

Key words: instrumentation: detectors - X-rays: general - space vehicles

\section{Introduction}

The study of the spectral and temporal behaviour of sources over a wide range of energies is of primary importance for the understanding of the mechanisms that, in several instances, produce spectral features localized in different regions of the electromagnetic spectrum. This fact has become more and more evident in the last years, when the efforts made by several scientists to organize

Send offprint requests to: L. Piro: saxsci@alpha1.ias.fra.cnr.it complex campaigns of simultaneous observations with different satellites and ground-based facilities have been rewarded by excellent results.

BeppoSAX, $\mathbf{S}$ atellite italiano per $\mathbf{A}$ stronomia $\mathbf{X}$, is the first X-ray mission with a scientific payload covering more than three decades of energy - from 0.1 to $300 \mathrm{keV}$ - with a relatively large area, a medium energy resolution and imaging capabilities (resolution of about 1.5) in the range of $0.1-10 \mathrm{keV}$. This capability, in conjunction with the presence of wide field instruments primarily aimed at discovering transient phenomena, which could then be observed with the wide band instruments, will provide an unprecedented opportunity to study the wide band behaviour of several classes of X-ray sources.

BeppoSAX is a major program of the Italian Space Agency (ASI) with participation of the Netherlands Agency for Aereospace Programs (NIVR). The mission development is supported by a consortium of scientific institutes, The BeppoSAX Consortium, composed as follows:

- Istituto Astrofisica Spaziale (IAS), C.N.R., Frascati and Unita' GIFCO Roma

- Istituto di Fisica Cosmica ed Applicazioni Informatica (IFCAI) and Unita' GIFCO, Palermo

- Istituto Fisica Cosmica e Tecnologie Relative (IFCTR), CNR and Unita' GIFCO, Milano

- Istituto per le Tecnologie e Studio Radiazioni Extraterrestri (ITeSRE), C.N.R., Bologna and Universita' di Ferrara

- Space Research Organization of the Netherlands (SRON), The Netherlands

- Space Science Department (SSD), ESA, Noordwijk, The Netherlands.

The Max Planck Institute for Extraterrestrial Physics has supported the tests and calibrations of the X-ray optics and the focal plane detectors. 
Prime industrial contractors are Alenia Spazio for the Space Segment and Nuova Telespazio for the Ground Segment.

The structure of this paper is as follows: in Sect. 2 we illustrate the satellite and scientific instruments; in Sect. 3 we describe the operations; in Sect. 4 we show the scientific capabilities of the instruments and in Sect. 5 we illustrate the main scientific objectives and the program of the mission ${ }^{1}$.

\section{The satellite and the scientific instruments}

\subsection{The scientific payload}

The configuration of the scientific payload and the energy bands covered by the different instruments are presented in Fig. 1 and Fig. 2 respectively. The wide band capability is provided by a set of instruments co-aligned with the $Z$ axis of the satellite (Fig. 1), Narrow Field Instruments (hereafter NFI) and consisting of (Table 1):

- MECS (Medium Energy Concentrator Spectrometers): a medium energy $(1.3-10 \mathrm{keV})$ set of three identical grazing incidence telescopes with double cone geometry (Citterio et al. 1985; Conti et al. 1994), with position sensitive gas scintillation proportional counters in their focal planes (Boella et al. 1996 and references therein).

- LECS (Low Energy Concentrator Spectrometer): a low energy $(0.1-10 \mathrm{keV})$ telescope, identical to the other three, but with a thin window position sensitive gas scintillation proportional counter in its focal plane (Parmar et al. 1996 and references therein).

- HPGSPC, a collimated High Pressure Gas Scintillation Proportional Counter $(4-120 \mathrm{keV}$, Manzo et al. 1996 and references therein).

- PDS, a collimated Phoswich Detector System $(15-300 \mathrm{keV}$, Frontera et al. 1996 and references therein).

Perpendicular to the axis of the NFI and pointed in opposite directions there are two coded mask proportional counters (Wide Field Cameras, WFC, Jager et al. 1996 and references therein) that provide access to large regions of the sky in the range $2-30 \mathrm{keV}$. Each WFC has a field of view of $20^{\circ} \times 20^{\circ}$ (FWHM) with a resolution of $5^{\prime}$.

Finally, the four lateral active shields of the PDS will be used as monitor of gamma-ray bursts with a fluence greater than about $10^{-6} \mathrm{erg} \mathrm{cm}^{-2}$ in the range $60-600 \mathrm{keV}$, with a temporal resolution of about $1 \mathrm{~ms}$.

\footnotetext{
1 On-line information on the mission can be obtained via WWW at any of the following URLs: http://www.sdc.asi.it/sax_main.html http://www.ias.fra.cnr.it/ias-home/sax/sax_main.html http://www.tesre.bo.cnr.it/Sax/sax_main.html http://astro.estec.esa.nl/SAgeneral/Research/Sax/mirror/sax_main.html
}

Each instrument (the four NFI and the two WFC's) is controlled by a dedicated computer which has, in particular, the task of performing on board pre-processing of the scientific data according to the acquisition mode required by the specific observation. The basic mode is the Direct mode, in which each single event is transmitted with the full information. For sources fainter than about $0.3 \mathrm{Crab}\left(\simeq 10^{-8} \mathrm{erg} \mathrm{cm}^{-2} \mathrm{~s}^{-1}\right.$ in the $2-10 \mathrm{keV}$ range), the telemetry (see next Section) is sufficient to support this mode for all instruments simultaneously. For brighter sources Indirect modes allow to reduce the telemetry by producing on-board spectra, images and light curves with a large choice of parameters. The scientific data packets produced by each instrument are received by the main on-board processor (see next Section) on the basis of the overall telemetry occupation and instrument priority programmable from ground.

\subsection{The spacecraft and the other subsystems}

The main characteristics of the spacecraft are presented in Table 2. BeppoSAX is a three axes stabilized satellite, with a pointing accuracy of $1^{\prime}$. The main attitude constraint derives from the need to maintain the normal to the solar arrays within $30^{\circ}$ from the Sun, with occasional excursions to $45^{\circ}$ for some WFC observations. Due to the low orbit (see next section) the satellite will be in view of the ground station for only a limited fraction of the time. Data will be stored onboard on a tape unit with a capacity of 450 Mbits and transmitted to ground every orbit during station passage. The average data rate available to instruments is about $60 \mathrm{kbit} / \mathrm{s}$, but peak rates of up to $100 \mathrm{kbit} / \mathrm{s}$ can be retained for part of each orbit.

With the solar panels closed, the spacecraft is $3.6 \mathrm{~m}$ in height and $2.7 \mathrm{~m}$ in diameter. The total mass amounts to $1400 \mathrm{~kg}$, with a payload of $480 \mathrm{~kg}$. The structure of the satellite consists of three basic functional subassemblies:

- the Service Module, in the lower part of the spacecraft, which houses all the subsystems and the electronic boxes of the scientific instruments;

- the Payload Module, which houses the scientific instruments and the Star trackers;

- the Thermal Shade Structure, that encloses the Payload Module.

The primary sub-systems of the satellite are:

- The Attitude Orbital Control System (AOCS), that performs attitude determination and manoeuvres and operates the Reaction Control Subsystem in charge of orbit recovering. It includes redundant magnetomers, Sun acquisition sensors, three star trackers, six gyroscopes (three of which are for redundancy), three magnetic torquers and four reaction wheels, all controlled by a dedicated computer. The AOCS ensures a pointing accuracy of $1^{\prime}$ during source observations and manoeuvres with a slew rate of $10^{\circ}$ per min. 


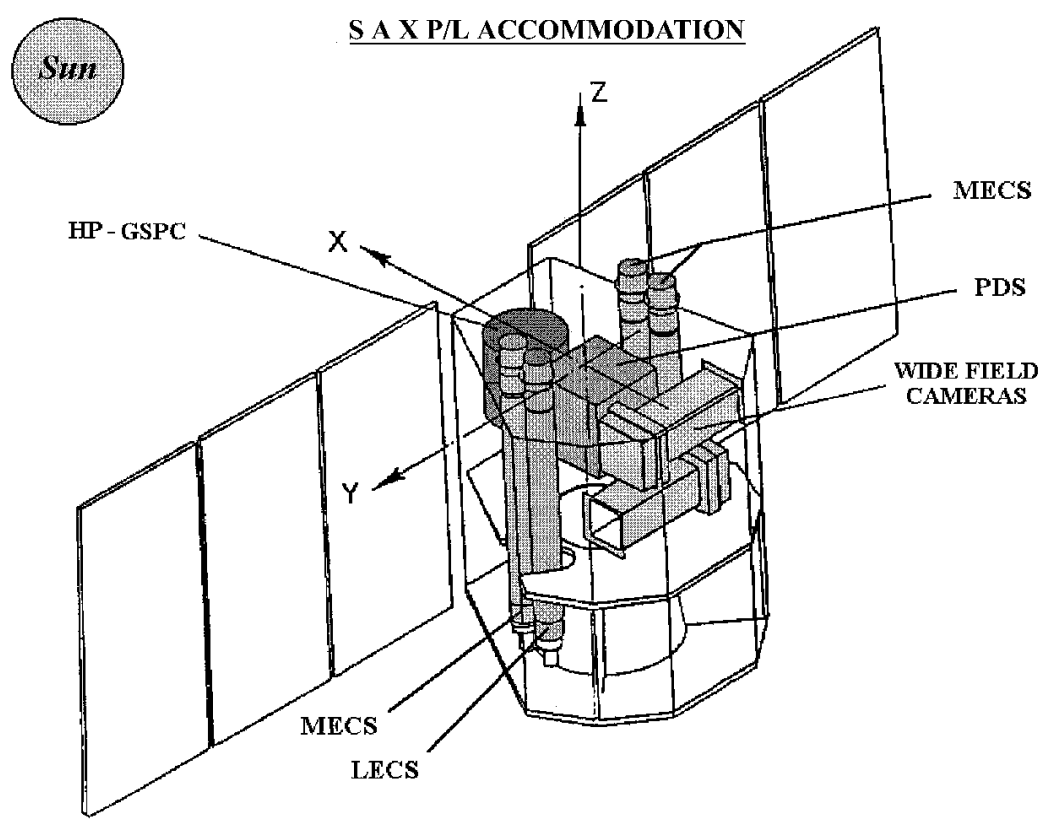

Fig. 1. BeppoSAX scientific payload accommodation

Table 1. BeppoSAX instruments

\begin{tabular}{|c|c|c|c|c|c|}
\hline Instrument & $\begin{array}{l}\text { Range } \\
(\mathrm{keV})\end{array}$ & $\begin{array}{l}\text { Field } \\
\left({ }^{\circ} \mathrm{FWHM}\right)\end{array}$ & $\begin{array}{l}\text { Angul. Res. }{ }^{(3)} \\
(\text { arcmin })\end{array}$ & $\begin{array}{l}\text { Area } \\
\left(\mathrm{cm}^{2}\right)\end{array}$ & $\begin{array}{l}\text { Energy Res. } \\
(\% \text { FWHM })\end{array}$ \\
\hline $1 \operatorname{LECS}^{(1)}$ & $0.1-10$ & 0.5 & $3.5 @ 0.25 \mathrm{keV}$ & $22 @ 0.25 \mathrm{keV}$ & $8 \times(E / 6)^{-0.5}$ \\
\hline $3 \operatorname{MECS}^{(1)}$ & $1.3-10$ & 0.5 & $1.2 @ 6 \mathrm{keV}$ & $150 @ 6 \mathrm{keV}$ & $8 \times(E / 6)^{-0.5}$ \\
\hline $1 \mathrm{HPGSPC}^{(1)}$ & $4-120$ & 1.1 & collimated & 240@30 keV & $4 \times(E / 60)^{-0.5}$ \\
\hline $1 \mathrm{PDS}^{(1)}$ & $15-300$ & 1.3 & collimated & $600 @ 80 \mathrm{keV}$ & $15 \times(E / 60)^{-0.5}$ \\
\hline $2 \mathrm{WFC}^{(2)}$ & $2-30$ & $20 \times 20$ & 5 & $140 @ 10 \mathrm{keV}$ & $18 \times(E / 6)^{-0.5}$ \\
\hline
\end{tabular}

Note: ${ }^{(1)}$ Narrow Field Instruments (NFI); ${ }^{(2)}$ per unit through mask; ${ }^{(3)}$ radius containing $50 \%$ of the power.

- The On Board Data Handler (OBDH) is the core for data management and system control on the satellite and it also manages the communication interfaces between the satellite and the ground station. Its computer supervises all subsystem processor activities, such as those of each instrument, and the communication busses.

- Other subsystems are devoted to managing the power generated by the solar panels and battery energy storage (Electric Power and Solar Array Subsystem), to raising the orbit in case of decay below $450 \mathrm{~km}$ (Reaction Control Subsystem), to controlling the temperature of the satellite (Thermal Control Subsystem) and to ensure communication with the Ground Station (Telemetry Tracking and Command Subsystem).
Table 2. BeppoSAX: spacecraft main characteristics

\begin{tabular}{ll}
\hline Dimensions: diameter & $2.7 \mathrm{~m}$ (sol. panels closed) \\
height & $3.6 \mathrm{~m}$ \\
Mass: tot. & $1400 \mathrm{~kg}$ \\
payload & $480 \mathrm{Kg}$ \\
Power: tot. & $800 \mathrm{~W}$ \\
payload & $260 \mathrm{~W}$ \\
Sun angle constraint & $30^{\circ}\left(\operatorname{max~} 45^{\circ}\right)$ \\
Pointing & $3 \mathrm{axes} \mathrm{stabilized}$ \\
Pointing accuracy & $<1 \mathrm{arcmin}$ \\
Max slew rate & $10^{\circ} / \mathrm{min}$ \\
On board memory & $510 \mathrm{Mbit}$ \\
Telemetry to instruments & $\max 100 \mathrm{kbit} / \mathrm{s}$ (average 60) \\
Downlink recorded data & $917 \mathrm{kbit} / \mathrm{s}$ \\
\hline
\end{tabular}




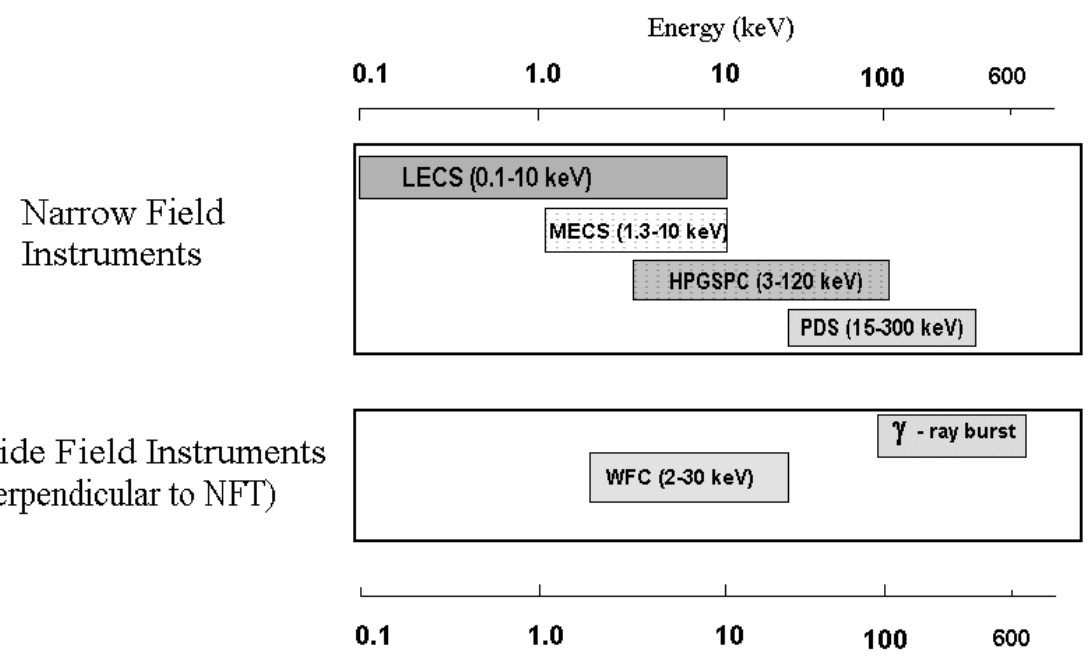

Fig. 2. Energy coverage of BeppoSAX instrument

Table 3. BeppoSAX: launch, orbit

\begin{tabular}{ll}
\hline Launch date & Apr. 30, 1996 \\
Vehicle & Atlas-Centaur \\
Circular Orbit at & $600 \mathrm{~km}$ \\
inclination & 3.9 \\
Orbital period & $96 \mathrm{~min}$ \\
n. orbit per day & 15 \\
contact duration & $10 \mathrm{~min}$. \\
Ground Station & Malindi, Kenya \\
expected lifetime & 2-4 years \\
\hline
\end{tabular}

\section{Ground operations}

BeppoSAX was launched on April 301996 by an AtlasCentaur directly into a $600 \mathrm{~km}, 96 \mathrm{~min}$ orbit at 3.9 inclination. The satellite will thus nearly avoid the South Atlantic Anomaly and take full advantage of the screening effect of the Earth's magnetic field in reducing the cosmic ray induced background, an aspect particularly relevant for high energy instruments (HPGSPC and PDS).

The satellite is operated directly from the Operation Control Center (OCC) in Rome, through a bidirectional (command transmission and telemetry data collection) Intelsat link between the OCC and the Telemetry and Telecommand (TT\&C) station located on the equator at the Italian base near Malindi (Kenya). The satellite passes over the TT\&C station every orbit for a contact period of 10 minutes. During each pass the Ground Station performs the following activities: telecommand up-linking; mass memory downloading; spacecraft doppler and ranging; spacecraft time sinchronization.

The OCC is the core of the satellite operational management with the following activities: telecommand generation and validation, orbit and attitude determination; short-term telemetry archiving; health monitoring of satellite and its sub-systems.

As part of the OCC, the Scientific Operation Center (SOC) will deal specifically with scientific operations: monitoring of payload parameters; quick-look analysis of scientific data in real time, aimed in particular to bright X-ray transients discovery and Target of Opportunity (TOO) alert; short and long term scheduling.

The Scientific Data Center (SDC) is located in Rome, at the same site as the OCC. Along with the Mission Scientist it is the main interface of the scientific community to the BeppoSAX project. Its main tasks are: collection and archiving of the proposals from the scientific community (Sect. 5.2); production of the list of observations from the approved proposals to be processed by the OCC for schedule generation; archiving of telemetry on optical disks; production and distribution of data $(\mathrm{FOT}=$ Final Observing Tape) to the observers; off-line analysis of quick look data for TOO follow up program's assessment; integration, development and maintainance of scientific software and calibrations along with the Institutes of the BeppoSAX Consortium; distribution of calibration data, software and general information about the mission; support to guest observers for data analysis and proposal preparation; set up of the results data base.

The Mission Scientist overviews all the scientific activities of the program, and in tight contact with the other scientific components, takes care in particular of: schedule of observations and TOO; calibrations of instruments; issue of Announcement of Opportunities and the technical description of the mission (Piro et al. 1995).

\section{Scientific capabilities of BeppoSAX instruments}

In Fig. 3 we show the effective area of the BeppoSAX instruments. Note that the effective area of the NFI is 
increasing with energy following a power law with positive slope. This will partially compensate for the spectral shape of the celestial objects, though not quite completely, because the HPGSPC and PDS are generally background dominated whereas MECS and LECS are source dominated (Fig. 4). A relevant property of the BeppoSAX scientific payload is the broad overlapping energy response of the various instruments, that will allow cross-calibration of the instruments.

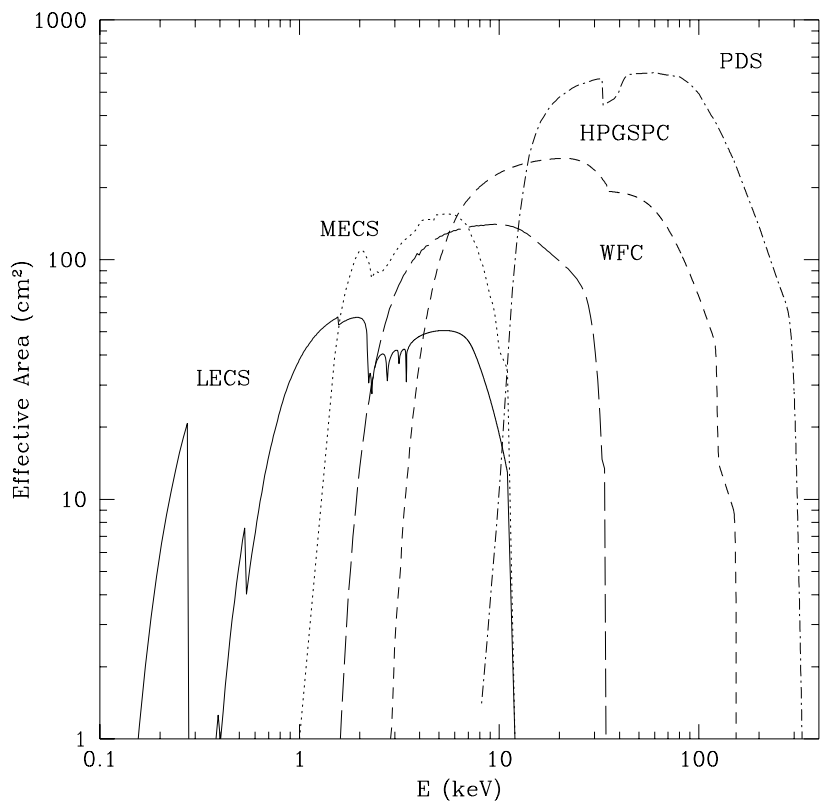

Fig. 3. Effective area of BeppoSAX instruments

\subsection{Scientific capabilities of the LECS and MECS}

The four X-ray optics with GSPC's detector in the focal plane have been designed to deliver:

- an effective area around $7 \mathrm{keV}$ sufficient to detect in $10^{5} \mathrm{~s}$ an iron line with an equivalent width of $150 / F_{\mathrm{X}}^{0.5}$ $\mathrm{eV}$ at $5 \sigma$ from a source with $F(2-10 \mathrm{keV})=F_{\mathrm{X}}$ $10^{-11} \mathrm{erg} \mathrm{cm}^{-2} \mathrm{~s}^{-1}$, down to $F_{\mathrm{X}} \simeq 0.1$, when the continuum level at $6.4 \mathrm{keV}$ becomes 2 times the background level.

- an energy resolution of $8 \%$ @ $6 \mathrm{keV}$ and $25 \%$ @ $0.6 \mathrm{keV}$, that below $0.6 \mathrm{keV}$ becomes comparable with that of a CCD (e.g. Tanaka et al. 1994);

- spectral coverage below the carbon edge $(0.3 \mathrm{keV})$, where the LECS detector will provide 2-3 independent energy bins;

- reasonable imaging capabilities (1.5 arcmin @ $6 \mathrm{keV})$. Combined with their energy resolution, they will perform spatially resolved spectroscopy of extended sources such as clusters of galaxies and supernova remnants. Furthermore they will do spectral measurements on weak point-like sources $\left(F(2-10 \mathrm{keV}) \simeq 10^{-13} \mathrm{erg}\right.$

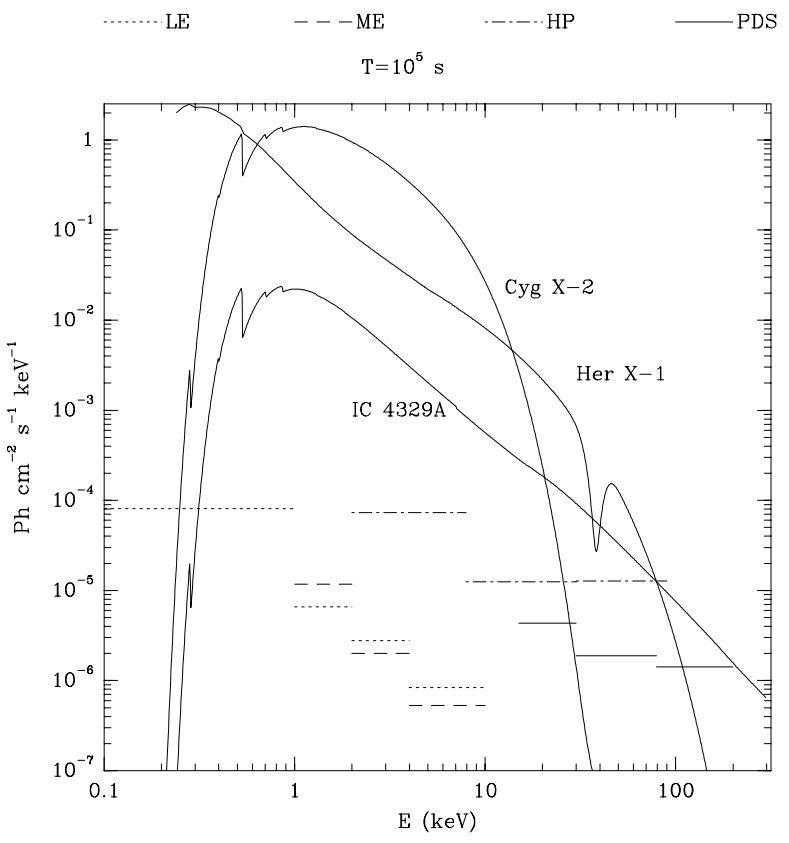

Fig. 4. Five sigma BeppoSAX NFI instrument sensitivity for a $100 \mathrm{ksec}$ exposure. For the HPGSPC and PDS it was assumed to point for half of the time off-source to measure the background. Spectra of representative galactic and extragalactic sources are shown

$\mathrm{cm}^{-2} \mathrm{~s}^{-1}$ ), thanks to the low background included in the small size of the source spot.

In Fig. 5 we show the capability of the combined LECS and MECS to perform spectral measurements of thin plasma spectra. Taking as reference a $30 \%$ error on the relevant parameters we see that the temperature can be determined down to $F(0.5-4.5 \mathrm{keV}) \simeq 210^{-13} \mathrm{erg}$ $\mathrm{cm}^{-2} \mathrm{~s}^{-1}$, the iron abundance down to $F(0.5-4.5 \mathrm{keV}) \simeq$ $10^{-12}$ erg $\mathrm{cm}^{-2} \mathrm{~s}^{-1}$ and the abundance of elements other than Hydrogen and Iron down to $F(0.5-4.5 \mathrm{keV}) \simeq$ $310^{-12} \mathrm{erg} \mathrm{cm}^{-2} \mathrm{~s}^{-1}$.

In case of sources with power law spectra, such as AGN, spectral index measurements can be carried out down to fluxes $\lesssim 10^{-13} \mathrm{erg} \mathrm{cm}^{-2} \mathrm{~s}^{-1}$, as shown in Fig. 6 . The typical spectrum that could be obtained for an AGN with $F(2-10 \mathrm{keV})=10^{-12}$ erg $\mathrm{cm}^{-2} \mathrm{~s}^{-1}$ in $10^{4} \mathrm{~s}$ is shown in Fig. 7.

An example of the capability of studying narrow features is shown in Fig. 8, where an OVII edge at $0.8 \mathrm{keV}$ and a broad iron line at $6.4 \mathrm{keV}(\mathrm{FWHM}=0.7 \mathrm{keV})$ in a typical Seyfert 1 galaxy are well resolved by the LECS and MECS respectively.

\subsection{Scientific capabilities of the HPGSPC and PDS}

The high energy instruments aboard BeppoSAX are respectively a high pressure (5 atm. Xe) GSPC and a scintillator (NaI/CsI) phoswich detector system. Several 


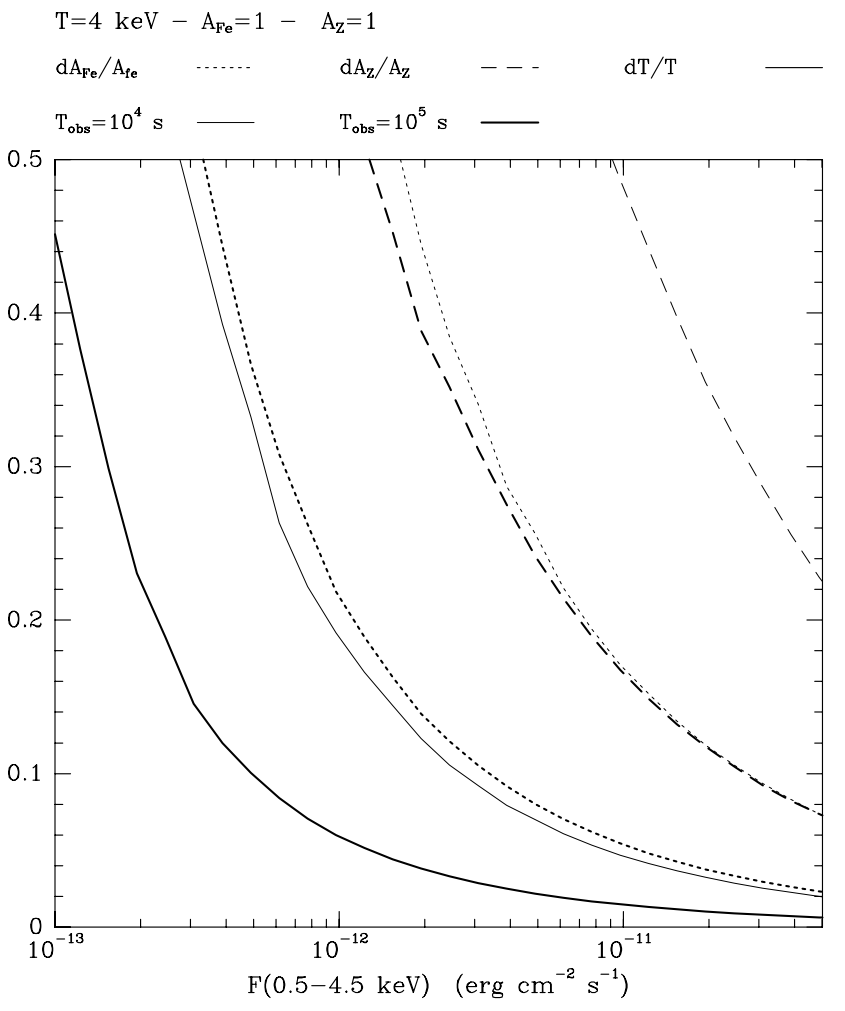

Fig. 5. Capability of LECS and MECS in measuring spectral parameters of thin plasma spectra. The relative error on the temperature (continuous lines), iron abundance (dotted lines) and lighter elements abundance (dashed lines) are shown as a function of the source flux for exposure times of $10^{4} \mathrm{~s}$ (thin lines) and $10^{5} \mathrm{~s}$ (thick lines)

solutions have been implemented to minimize systematic effects and the background in these collimated detectors:

- the background is monitored continuosly rocking the collimators on and off source with a typical period of one minute;

- the environmental background due to high energy particles is much lower than that of other current and near future missions, thanks to the low inclination orbit;

- the HPGSPC will be the first detector ever flown on a satellite to implement the technique of the fluorescence gate (Manzo et al. 1980) that will allow to decrease the background substantially above the Xenon edge (34 keV);

- both detectors have an active equalization system that will keep the gain within $0.5-0.25 \%$.

The PDS was specifically designed in order to minimize background so as to increase its sensitivity to background dominated sources (basically below $200 \mathrm{mCrab}$ ). From this point of view the expected performance should be comparable or better than that of the HEXTE detector aboard XTE (Bradt et al. 1993). XTE, on the other hand, remains superior for bright sources, given its larger area aimed to priviledge the timing information. The PDS sensitivity allows spectral measurements up to $200 \mathrm{keV}$ for sources of

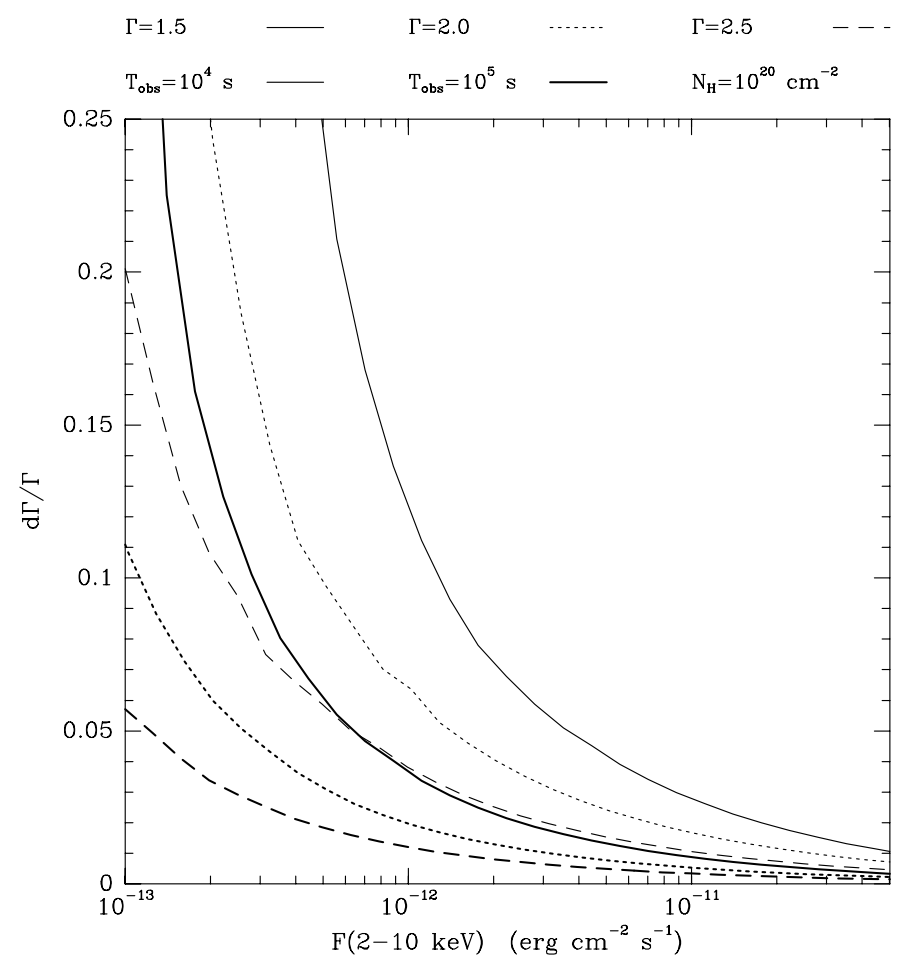

Fig. 6. Spectral index determination by the combined NFI. The relative error on the spectral index is given as a function of the flux for three values of the index $(\Gamma=1.5$ (continuous line), $\Gamma=2.0$ (dotted line), $\Gamma=2.5$ (dashed line) with $N_{H}=10^{20} \mathrm{~cm}^{-2}$ and two values of integration time ( $T=10^{4}$ s: thin lines, $T=10^{5}$ s: thick lines). Below $F \simeq 10^{-11}$ erg $\mathrm{cm}^{-2} \mathrm{~s}^{-1}$ the performances are primarily due to the LECS and MECS

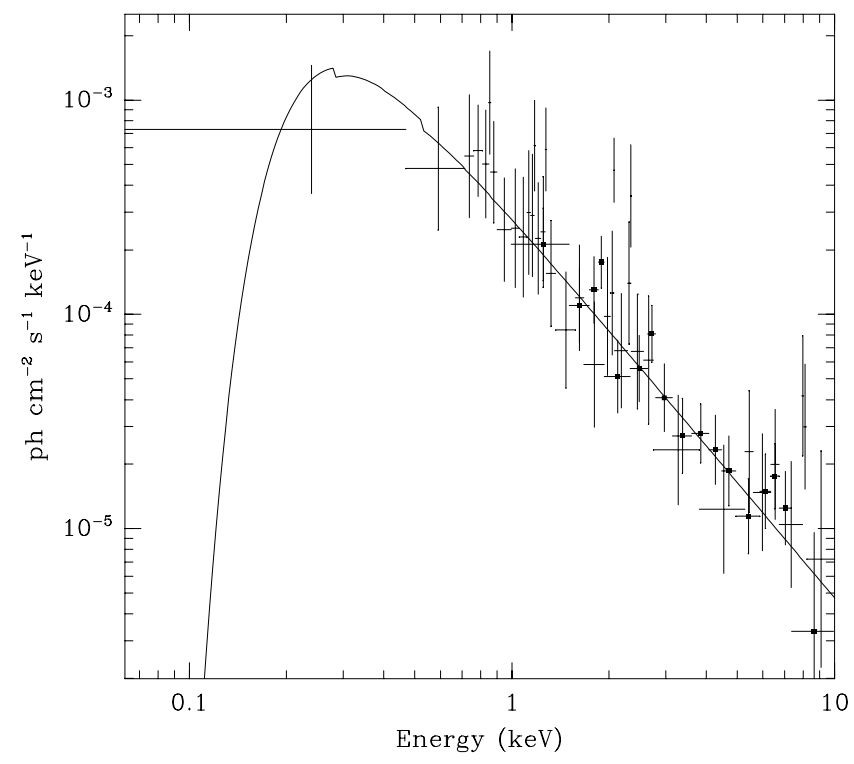

Fig. 7. Simulation of a spectrum of an AGN with $F(2-10 \mathrm{keV})=10^{-12} \mathrm{erg} \mathrm{cm}^{-2} \mathrm{~s}^{-1}$ observed by the LECS and MECS in $10^{4} \mathrm{~s}$ 


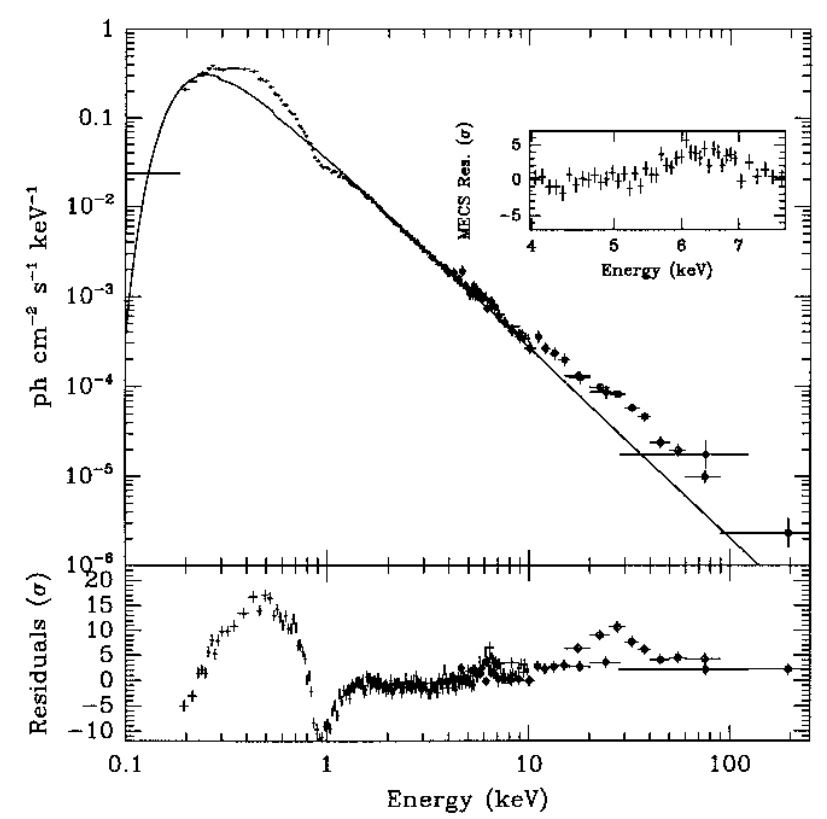

Fig. 8. Simulation of an observation by BeppoSAX-NFI (40000 s) of a Seyfert 1 galaxy (MCG-6-30-15) with several of the features observed in the last 10 years by different satellites: a soft excess below $1 \mathrm{keV}$ (EXOSAT), an edge of ionized oxygen at $0.8 \mathrm{keV}$ (ROSAT, ASCA), a broad iron line (FWHM = $0.7 \mathrm{keV}$ ) at $6.4 \mathrm{keV}$ and a high energy bump bewtween 10 and $200 \mathrm{keV}$ (GINGA). The spectrum is fitted with a simple power law and the residuals clearly show all these components. In the blow-up the residuals of the MECS around the broad iron line are plotted, showing that the line is well resolved by the detector

about $1 \mathrm{mCrab}\left(F(2-10 \mathrm{keV}) \simeq 210^{-11} \mathrm{erg} \mathrm{cm}^{-2} \mathrm{~s}^{-1}\right.$, Fig. 4).

The main scientific motivation that lead to the design of the HPGSPC and its inclusion in the payload is its unprecedented energy resolution, 4\%@60 keV, that will allow detailed line spectroscopy in hard X-rays. The spectroscopic capabilities of HPGSPC and PDS are illustrated in Fig. 9, that represents a simulation of a possible cyclotron absorption spectrum of a bright transient, X0331 + 53, a $\mathrm{X}$-ray pulsar. GINGA observed only the first harmonic and tentatively the second one (Makishima et al. 1990). The BeppoSAX high energy instruments can measure very well not only the first harmonic but also determine the possible presence of higher harmonics in a spectral range where the information is - up to now - missing. Pulse phase dependent spectroscopy will be easily achieved for such sources as well.

\subsection{Narrow field instrument combined scientific capabilities}

The sensitivity curves of BeppoSAX NFI are given in Fig. 4 along with spectra of some typical galactic and

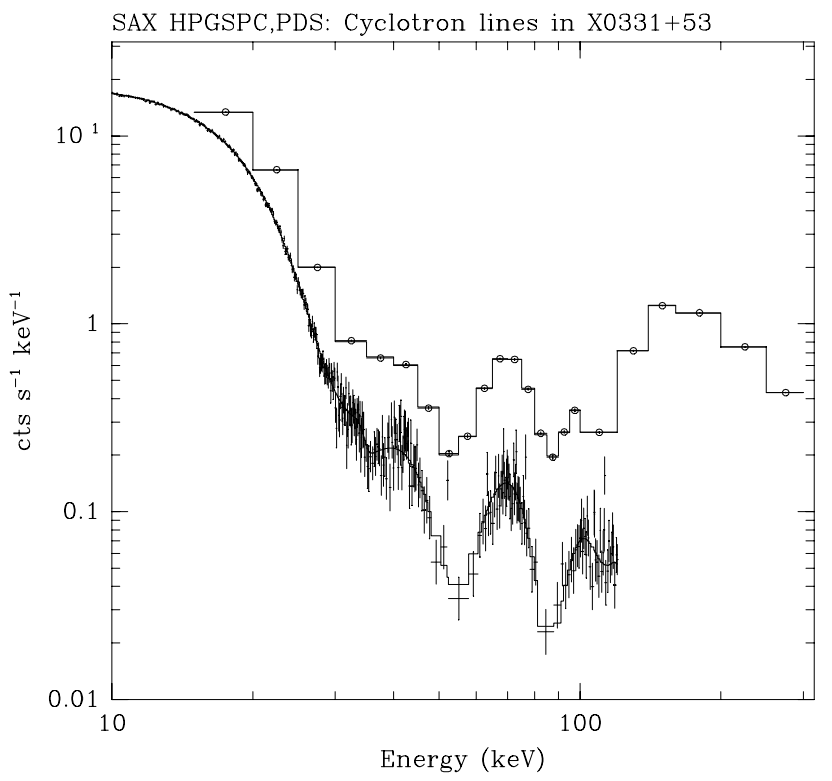

Fig. 9. Cyclotron lines in the transient X0331+53. The simulation shows a possible spectrum (four harmonics) of the object observed by the HPGSPC (crosses) and the PDS (circles) in $10000 \mathrm{~s}$. It clearly illustrates the capability of BeppoSAX of measuring such features also in the unexplored region above $40 \mathrm{keV}$

extragalactic sources. Basically for AGN-like spectra it is possible to determine the spectrum up to $200 \mathrm{keV}$ for sources down to about $1 \mathrm{mCrab}$. As an example in Fig. 8 we show the simulation of the combined BeppoSAX-NFI spectrum of a Seyfert 1 galaxy, namely MCG-6-30-15, with all the spectral component and features detected by several satellites in the past. Starting from the low energy part there is: a soft excess observed by EXOSAT (Pounds et al. 1986), an OVII edge around $0.8 \mathrm{keV}$ observed by ROSAT (Nandra \& Pounds 1992) and ASCA (Fabian et al. 1994), an iron line at $6.4 \mathrm{keV}$ and a high energy bump above $10 \mathrm{keV}$ detected by GINGA (Matsuoka et al. 1990). All these components can be measured with good accuracy with BeppoSAX in a single shot for the first time.

\subsection{Scientific capabilities of WFC}

The sensitivity of the WFC depends on the pointing direction in the sky, because each source in the field of view contributes to the overall background. Towards high-galactic latitude the Cosmic Diffuse X-Ray Background is the main contributor to the background. In this case the sensitivity is at the order of a few mCrab in $10^{4} \mathrm{~s}$ (Fig. 10). This will allow the monitoring of faint sources like AGN (Fig. 11), as well as their prime objective, which is the survey of the galactic plane and the search of X-ray transients for follow-up studies with the narrow field instruments.

On the basis of the $\log N-\log S$ distribution of gammaray burst and assuming $F_{\mathrm{X}}$ about $1 / 100$ of $F_{\gamma}$ we expect 
to detect a few X-ray counterparts to gamma-ray bursts per year, thus positioning the events within about $3^{\prime}$ and gathering broad band information with the simultaneous observation of the gamma-ray burst monitor.

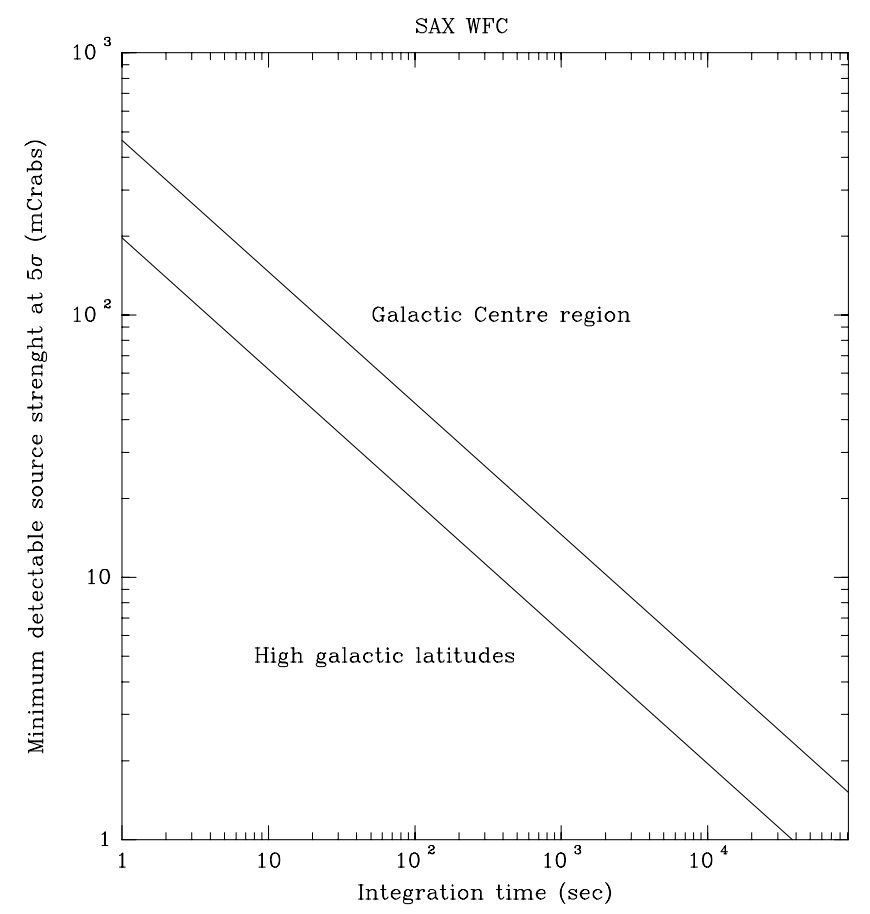

Fig. 10. Five sigma sensitivity of one WFC

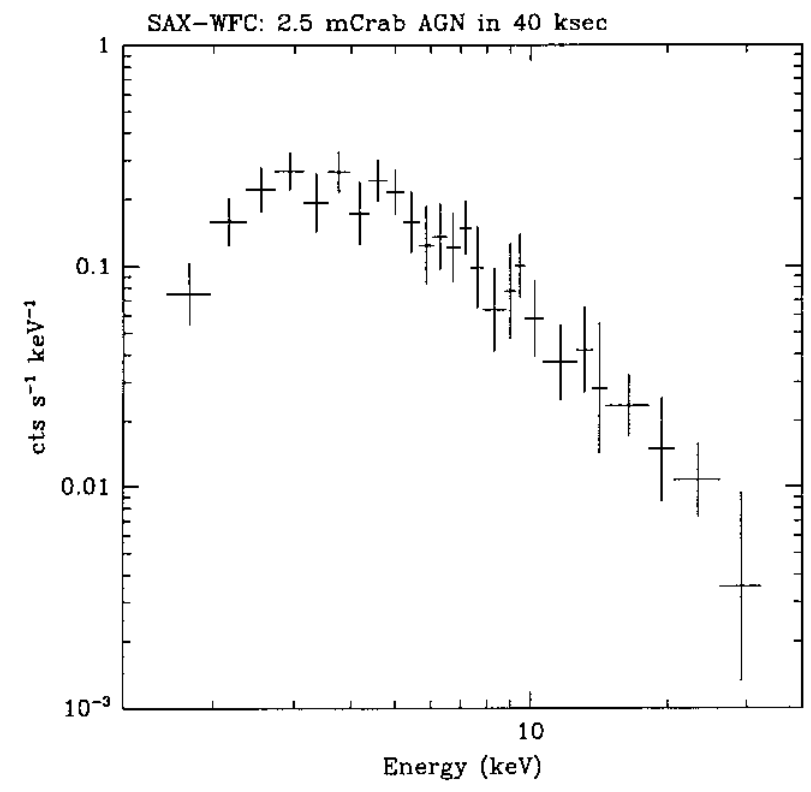

Fig. 11. The spectrum of a AGN (2.5 mCrab) observed by one WFC in $40000 \mathrm{~s}$

\section{Scientific program}

\subsection{Scientific objectives}

Given the instrument capabilities over the wide energy range described in the previous sections, BeppoSAX can provide an important contribution in several areas of X-ray astronomy (Perola 1990) such as:

- Compact galactic sources: Shape and variability of the various continuum components and of the narrow spectral features (e.g. iron line, cyclotron lines); phase resolved spectroscopy. Discovery and study of X-ray transients.

- Active Galactic Nuclei: spectral shape and variability of the continuum and of the narrow and broad features from 0.1 to $200 \mathrm{keV}$ in bright objects (soft excess, warm and cold absorption and related $\mathrm{O}$ and $\mathrm{Fe}$ edges, iron line and high energy bump, high energy cut-off); spectral shape and variability of different classes of AGN down to $1 / 20$ of $3 \mathrm{C} 273$ up to $100-200 \mathrm{keV}$; spectra of weak AGN (e.g. high redshift objects) up to $10 \mathrm{keV}$.

- Clusters of galaxies: spatially resolved spectra of nearby objects and the study of temperature gradients; chemical composition and temperature of more distant clusters.

- Supernova remnants: spatially resolved spectra of extended remnants; spectra of Magellanic Cloud remnants.

- Normal Galaxies: spectra from 0.1 to $10 \mathrm{keV}$ of the extended emission.

- Stars: multi-temperature spectra of stellar coronae from 0.1 to $10 \mathrm{keV}$; temperature/spatial structure of coronae by observation of eclipsing binary systems; evolution of flares.

- Gamma-ray bursts: temporal profile with 1 msec resolution from 60 to $600 \mathrm{keV}$. X-ray counterparts of a subset with positional accuracy of $5^{\prime}$.

\subsection{Strategy and operations}

Due to the constraint on the solar panel orientation (Sect. 2.2 ), the sky region accessible to the NFI at any time is a band $60^{\circ}$ wide $(50 \%$ of the sky, slightly larger for the WFC) in the direction perpendicular to the sun vector. In six months all the sky will be therefore accessible. Depending on target position, the observing efficiency will be limited to $50 \%$ on average by Earth eclipses (the Earth subtends an angle of about $130^{\circ}$ at $600 \mathrm{~km}$ ) and by passages skirting the edge of the South Atlantic Anomaly.

With a lifetime of two - four years, BeppoSAX will be able to perform $1000-2000$ pointings with durations from $10^{4} \mathrm{~s}$ to $10^{5} \mathrm{~s}$. The NFI will be the prime instruments most of the time. When the NFI perform their sequence of pointed observations, the WFC will be operated in parallel (secondary mode) to point preferentially towards the galactic plane, to catch X-ray transients, or to monitor selected regions of the sky. We expect to detect about 
$10-20$ bright X-ray transients per year during the WFC observations. The observing program will be held flexible in order to accommodate these TOO for follow-up observations with the NFI, and the operational capability of BeppoSAX will allow an unexpected new target to be acquired within half a day of its discovery.

Thanks to their large field of view and the distribution of the NFI targets in the sky we expect that, in addition to the Galactic plane, a large fraction of the high Galactic latitude sky will also be covered by the WFC with a sensitivity of a few mCrabs.

The observing time of BeppoSAX is allocated on the basis of proposals submitted in responce to announcements of opportunity and selected by a Time Allocation Committee (TAC) under the auspices of the BeppoSAX Scientific Steering Committee. The observing time is divided in two parts. The first part is devoted to a Core Program amounting to $80 \%$ in the first round of 12 months, to $60 \%$ the second round of 12 months and $50 \%$ in the following. The Core Program will result from the selection of those proposals explicitely submitted for consideration as a part of it and that should be primarily devoted to systematic studies with particular regard to scientific objectives that exploit BeppoSAX capabilities. The Core Program observing time is reserved for competition only to proposals led by Principal Investigators (PI) belonging to Italian or Dutch institutions, to SSD or to the Max Planck Institute for Extraterrestrial Physics in Garching. The second part of the observing time, the Guest Observers Program, is open for competition to proposals led by PI from any country worldwide.

More details on the observing program as well as a detailed technical description of the mission can be found in the BeppoSAX Observers' Handbook (Piro et al. 1995) and via network (Sect. 1).

Acknowledgements. The BeppoSAX program is the result of the efforts of many entusiastic people and it is impossible to thank them all here. A particular tribute to the memory of the project manager Massimo Casciola, who gave so much to the mission, not only with his professional contributions and competence but also with his humanity. We acknowledge the key contribution to the mission by G. Di Cocco, B. Taylor, by the ASI team G. Manarini and B. Negri and R. Ibba, by NIVR, in particular A. Hoeke, by M. Palenzona of ESTEC, and by all the scientists and technical staff of the Consortium institutes and SDC, in particular L. Chiappetti, G. Conti, E. Costa, D. Dal Fiume, F. Favata, M. Feroci, F. Fiore, F. Frontera, S. Giarrusso, P. Giommi, P. Grandi, M. Guainazzi, J. Heise, R. Jager, M.C. Maccarone, G. Manzo, G. Matt, T. Mineo, S. Molendi, L. Nicastro, M. Orlandini, A. Parmar, S. Re, B. Sacco, A. Santangelo, M. Trifoglio. We would like to thank the teams in Alenia Spazio, in particular B. Strim, G. Finocchiaro, P. Santoro, P. Attina', W. Cugno, A. Martelli, in Nuova Telespazio, in particular M. Manca, L. Bruca, L. Cicali, L. Salotti, A. Coletta, G. Tarchini, C. De Libero and in Laben, in particular M. Iacopini, F. Volpi, A. Lenti, E. Alippi, F. Monzani, G. Falcetti, for their professional job on the satellite, the ground segment, the payload. Thanks are due to the team of the Lockheed Martin, in particular B. Sherwood, for the succesful launch. The key role of O. Citterio in the optics design is acknowledged.

\section{References}

Boella G., et al., 1996, A\&A (this volume)

Citterio O., et al., 1985, SPIE Proc. 597, 102

Conti G., et al., 1994, SPIE Proc. 2279, 101

Fabian A., et al., 1994, PASJ 46, L59

Frontera, et al., 1996, A\&A (this volume)

Jager R., et al., 1996, A\&AS (in press)

Makishima, et al., 1990, ApJ 365, L69

Manzo G., Davelaar J., Peacock A., et al., 1980, Nucl. Instr. Meth. A 177, 603

Manzo G., et al., 1996, A\&A (this volume)

Matsuoka M., Piro L., Yamauchi M., Makishima M., 1990, ApJ 361,440

Nandra P., Pounds K., 1992, Nat 359, 215

Parmar A., et al., 1996, A\&A (this volume)

Perola G.C., 1990, Adv. Space Res. 10, 295

Piro L., et al., 1995, SAX Observers' Handbook, issue 1.0

Pounds K., et al., 1986, MNRAS 221, 7

Tanaka Y., Inoue H., Holt S.S., 1994, PASJ 46, L37 